\title{
Previous spatial learning is required for the development of place preference in rats following chronic administration of and withdrawal from morphine
}

\author{
Javier J.L. Rico, Vanessa M. Castilho, Sílvio Morato and Manoel Jorge Nobre \\ Universidade de São Paulo, Ribeirão Preto, Brazil
}

\begin{abstract}
The positive reinforcing properties of addictive drugs have a primary role in the development of drug dependence. In the field of opiates, great attention has been given to this phenomenon, as well as to the negative properties eliciting craving and inducing relapse during withdrawal. This study was designed to evaluate whether elevated plus-maze (EPM) experienced rats withdrawn from low doses of subcutaneous (s.c.) morphine $(10 \mathrm{mg} / \mathrm{kg})$, in which a high anxiety level is the most prominent withdrawal symptom, acquire place preference when submitted to a conflict paradigm in which drug effects are paired with an aversive context: the distal part of the open arms of an EPM. Both the anxiety test and place preference conditioning were measured in the same apparatus, a biased version of the plus-maze. In order to verify the influence of previous EPM spatial learning on the performance of morphine-withdrawn rats, half the animals in this study experienced the EPM prior to treatment. Additional groups were also tested under the influence of morphine effects. The effects of the treatments were quantified through the analysis of three types of measures: anxiety was inferred from the use of the conventional measures (percentage of entries and time spent in the open-arms) and risk-assessment behaviours (frequency of stretched-attending postures - SAP, and time spent at the centre of the maze). Place preference conditioning was evaluated through analysis of the number of entries, total time spent and distance run in the open-arm extremities, which is where the animals were conditioned. The number of closed-arm entries was taken as an index of locomotor activity. Our results showed that (i) EPM naïve rats pre-treated with morphine did not develop place preference, behaving like control rats; (ii) rats that had previous experience in the EPM showed no changes in open-arm avoidance on the second exposure, when compared with rats naïve for this condition; (iii) previous spatial learning of the EPM contextual cues was, in fact, a requirement for anxiety-inducing place preference for the openarms in morphine-withdrawn rats and, (iiii) conditioned place preference was achieved both in rats under the effects of morphine and in withdrawal, probably through the influence of the positive or negative reinforcing effects promoted by the presence or absence of the drug in the central nervous system. Keywords: drug abuse, opiate withdrawal, anxiety, place-preference, elevated plus-maze.
\end{abstract}

Received 17 October 2008; received in revised form 3 February 2009; accepted 7 February 2009. Available online 29 June 2009

\section{Introduction}

It has been suggested that the positive reinforcing properties of addictive drugs have a primary role in the development of drug dependence (Koob \& Kreek, 2007; Wise, 2002; Wise \& Bozarth, 1987). In the field of opiates, since physical dependence appears later than the positive motivational effects during chronic opiate exposure, great attention has been given to both

Javier J.L. Rico, Vanessa M. Castilho and Sílvio Morato - Laboratório de Psicobiologia, Universidade de São Paulo FFCL- Ribeirão Preto, SP, Brazil. Manoel Jorge Nobre-Instituto de Neurociências \& Comportamento, Universidade de São Paulo - Ribeirão Preto, SP, Brazil, 14040-901. Corresponding regarding this article should be directed to Manoel Jorge Nobre, Instituto de Neurociências \& Comportamento, Universidade de São Paulo - Ribeirão Preto, SP, Brazil, 14040-901. Phone: (55 16) 3602-3788. E-mail: mjnes@usp.br;. the positive opiate-reinforcing properties that promote substance abuse and the negative reinforcing effects that elicit craving and induce relapse during withdrawal (Koob \& Kreek, 2007). Nonetheless, since the seminal study of Himmelsbach (1943), the withdrawal syndrome from opiates has mainly been linked with the negative affective states that precede severe physical disturbances after the chronic opiate treatment is broken, despite the craving associated with the attempt to avoid withdrawal-induced dysphoria. In this respect, we have previously shown that withdrawal from morphine elicits intense unconditioned fear reactions accompanied by significant Fos-protein immunoreactivity in brainstem areas that are known to regulate the expression of defensive behaviours in animals facing fear-provoking stimuli (Castilho, Borelli, Brandao, \& Nobre, 2008). In another study, we showed that withdrawal from low doses of morphine, which has the ability to promote slight physical symptoms in rats, increases fear reactions 
induced by the electrical stimulation of the dorsal aspects of the periaqueductal gray (Ávila et al., 2008).

As revealed by the study of Holmes \& Rodgers (1999), on initial exposure to the elevated plus-maze (EPM), animals acquire information about the entire spatial layout of the test and not only about the location of the more aversive open arms. With regard to this point, their subsequent responses are guided largely by an awareness of the location of the relatively safe closed arms. This could be one of the reasons why a second exposure to the EPM has the ability to inhibit open arm exploration (File, Zangrossi, Viana, \& Graeff, 1993). In the field of drug abuse, however, drug cues could change the preference for the safe place for one that signals drug delivery in animals submitted to a conflicting place-conditioning procedure in which a withdrawn animal learns to associate a non-safe region with the positive reinforcing effects of the drug.

In order to go one step further in this matter, this study was designed to evaluate whether EPM-experienced rats withdrawn from low doses of morphine, in which a high anxiety level is the most prominent withdrawal symptom, acquire place preference when submitted to a conflict paradigm in which drug effects are paired with an aversive context: the distal part of the open arms of an EPM. Therefore, for our purposes, both the anxiety test and place preference conditioning were measured in the same apparatus, a biased version of the plus-maze. In order to verify the influence of previous EPM spatial learning on the performance of morphine-withdrawn rats, half the animals in this study experienced the EPM immediately before the beginning of the treatment. Additional groups were also tested under the influence of morphine.

\section{Methods}

\section{Subjects}

Male Wistar rats from the animal house of the University of São Paulo in Ribeirão Preto, weighing 220$250 \mathrm{~g}$ at the beginning of the experiment, were used. They were housed in groups of four in Plexiglas-walled cages lined with wood shavings that were changed every three days. The animals were kept in a 12:12 dark/light cycle (lights on at 07:00 h) at $24 \pm 1^{0} \mathrm{C}$ and given free access to food and water. Before treatment, the animals had a threeday habituation period. The experiments reported in this article were approved by the local Ethics Committee and were performed in compliance with the recommendations of the Brazilian Society for Neuroscience and Behaviour which, in turn, are in accordance with the rules of the U. S. National Institutes of Health Guide for Care and Use of Laboratory Animals.

\section{Induction of morphine dependence and withdrawal}

Rats withdrawn from a chronic regimen of elevated doses of morphine show severe physical alterations (Himmelsbach, 1943) that could disguise the expression of affective reactions. In fact, withdrawal from opiates in rodents is characterized by the expression of a constellation of serious somatic signs such as jumping, paw tremor, weight loss, diarrhoea and wet dog shake (Bozarth \& Wise, 1984; Gellert \& Holtzman, 1978; Maldonado, Stinus, Gold, \& Koob, 1992). However, Papaleo and Contarino (2006) showed that mice submitted to a sixday period of increasing morphine doses (i.p, $10-50 \mathrm{mg} /$ $\mathrm{kg}$ ) and tested after a 32-hour withdrawal showed no differences in global opiate physical withdrawal scores when compared with their control groups. Thus, for our purposes, morphine dependence was obtained by injecting the drug subcutaneously (s.c., $10 \mathrm{mg} / \mathrm{ml} / \mathrm{Kg}$ ) twice a day (08:00 and 18:00 h) for 10 days. This is a low dose with the ability to selectively promote affective signs of withdrawal while avoiding the physical ones after treatment interruption (Ávila et al., 2008). The last injection was given on the morning of the $10^{\text {th }}$ day. Throughout the experiment the animals were gently handled on a daily basis for 3 minutes immediately before drug administration in order to reduce the stressful effects of the injection procedure. Tests were carried out 2 or 48 hours after the last control or drug injections (2- or 48-h groups, respectively). Independent groups of animals were used for each experiment.

\section{Drugs}

Morphine sulphate (Cristalia, Brazil) was prepared for injection in physiological saline $(0.9 \%)$ and all injections were made subcutaneously in a volume of $1 \mathrm{ml} / \mathrm{kg}$ body weight. The dose used in this study was based upon prior work on spontaneous opioid withdrawal (Avila et al., 2008).

\section{Elevated plus-maze}

In order to study the role of the negative reinforcing effects elicited in rats under morphine withdrawal in the induction of place preference, both the anxiety test and place preference conditioning were measured in the same apparatus, a biased version of the plus-maze, in a procedure adapted from the study of Salome et al. (2004). In addition, to verify the influence of a second exposure on conditioning, naïve rats or those previously experienced in the EPM were used.

The EPM apparatus was made of wood and consisted of four arms of equal dimensions (50 x 12 $\mathrm{cm})$. Two arms were enclosed by $40-\mathrm{cm}$ high walls and were arranged perpendicularly to two open arms. The structure was located inside an insulated room with 20 lux of luminosity at the end of the open arms, and was elevated $50 \mathrm{~cm}$ above the floor, with a $1-\mathrm{cm}$ Plexiglas rim surrounding the open arms to prevent falls (Pellow, Chopin, File, \& Briley, 1985). To allow openarm conditioning, a $12 \times 20-\mathrm{cm}$ Plexiglas wall divided the open arms into two main sections, referred to as proximal and distal (in relation to the central section). 
The animals were confined to these distal parts of the maze. This procedure was adapted from the study of Salome et al. (2004). The tests were conducted 2 or 48 hours after the last saline or morphine administration. During the pre-exposure drug (2-h) or withdrawal (48-h) sessions, the animals were placed in the centre of the EPM facing one of the closed arms and their behaviour was recorded on video for five minutes.

\section{Experimental procedures}

Two main groups were formed: animals with no previous experience in the plus-maze (EPM-naïve) and those previously exposed to the EPM before the start of chronic drug treatment (EPM-experienced). Each group was subdivided and all the animals were conditioned during 30 minutes a day for 10 days to the distal parts of the open arms of the plus-maze, immediately after either saline or morphine injections. The animals were exposed daily to one of the two open arms of the EPM immediately after each morning injection. This procedure allowed us to expose the animals to each open arm the same number of times, counterbalancing the place preference induced during conditioning. Finally, the saline and morphine groups were further subdivided into two groups and the animals were tested either under the effect of the drug (2-h group) or after 48 hours of withdrawal (48-h group). Thus, eight independent groups were formed: 2-h saline naïve rats $(\mathrm{n}=9), 48$-h saline naïve rats $(\mathrm{n}=10), 2$-h morphine naïve rats $(\mathrm{n}=9), 48$-h morphine naïve rats $(\mathrm{n}=10), 2$-h saline experienced rats $(\mathrm{n}=9), 48$-h saline experienced rats $(\mathrm{n}=10), 2$-h morphine experienced rats $(\mathrm{n}=9)$, and 48-h morphine experienced rats $48 \mathrm{~h}(\mathrm{n}=10)$.

\section{Behavioural measures}

The effects of the treatments were quantified through the analysis of three types of measures: anxiety was inferred from the use of conventional measures (percentage of entries and time spent in the open arms) and risk-assessment behaviour (frequency of stretched-attending postures - SAP, and time spent in the centre of the maze) (Cruz, Frei, \& Graeff, 1994; Pellow et al., 1985; Rogers \& Johnson, 1995). SAP was defined as an exploratory posture in which the body of the animal is stretched forward and then retracted to the original position without any forward locomotion (Holmes \& Rodgers, 1999). Place preference conditioning was evaluated through the analysis of the number of entries, total time spent and distance run in the open-arm extremities, which was where the animals were conditioned. The number of closed-arm entries was taken as an index of locomotor activity (Rodgers \& Johnson, 1995). Behaviour in the plus-maze was recorded with a camera (Everfocus, USA) linked to a monitor and video cassette recorder placed outside the experimental room.

\section{Statistical analysis}

The results are presented as means \pm SEM. The data obtained in each of the experiments were analyzed by means of a three-way factorial ANOVA with the EPM training (naïve $\mathrm{x}$ experienced), treatment (saline $\mathrm{x}$ morphine), and condition during the test (under drug effects (2-h) x 48-h drug withdrawal) as the main factors. Tukey HSD post-hoc comparisons were carried out whenever significant overall F-values were obtained. In all cases a probability level of $\mathrm{p}$ $<.05$ was considered significant.

\section{Results}

Figure 1 shows the percentage of time spent in the open arms (top) and the number of open- (middle) and

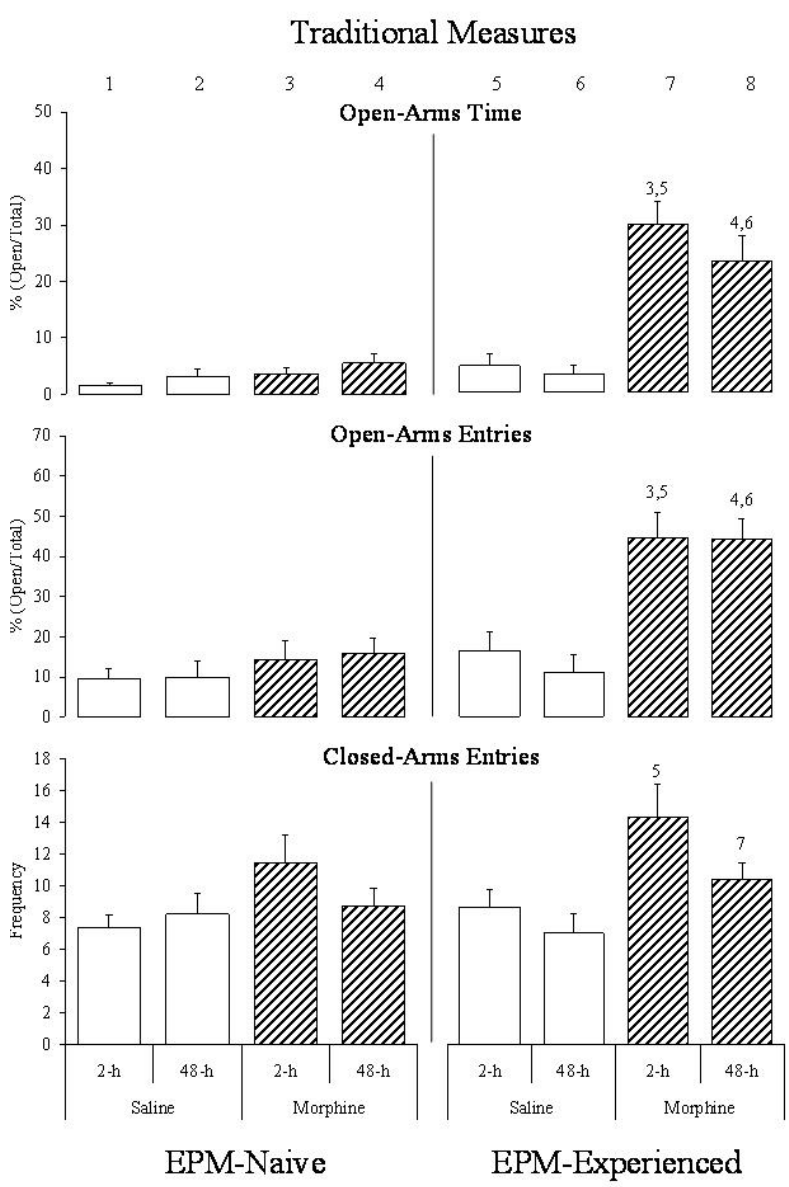

Fig. 1

Figure 1. Traditional Measures, as indices of anxiety, are presented as Means \pm SEM. Histograms represent the mean percentage of time spent in the open arms (top), percentage of open arms entries (middle), and number of closed arms entries (bottom) of rats not exposed to (EPM-naïve - left) or with previous experience (EPMexperienced - right) in the EPM, confined daily, during 10 days, to the distal parts of the open-arms immediately after saline or morphine s.c. injections. The animals were tested 2- (2-h) or 48hours (48-h) after the last injection. Three-way factorial ANOVA were carried-out, followed, when appropriate, by post-hoc Tukey HSD test; $p \leq .05$. Each column received a number ( 1 to 8 ) in order to depict the significant differences, as observed above histograms. 


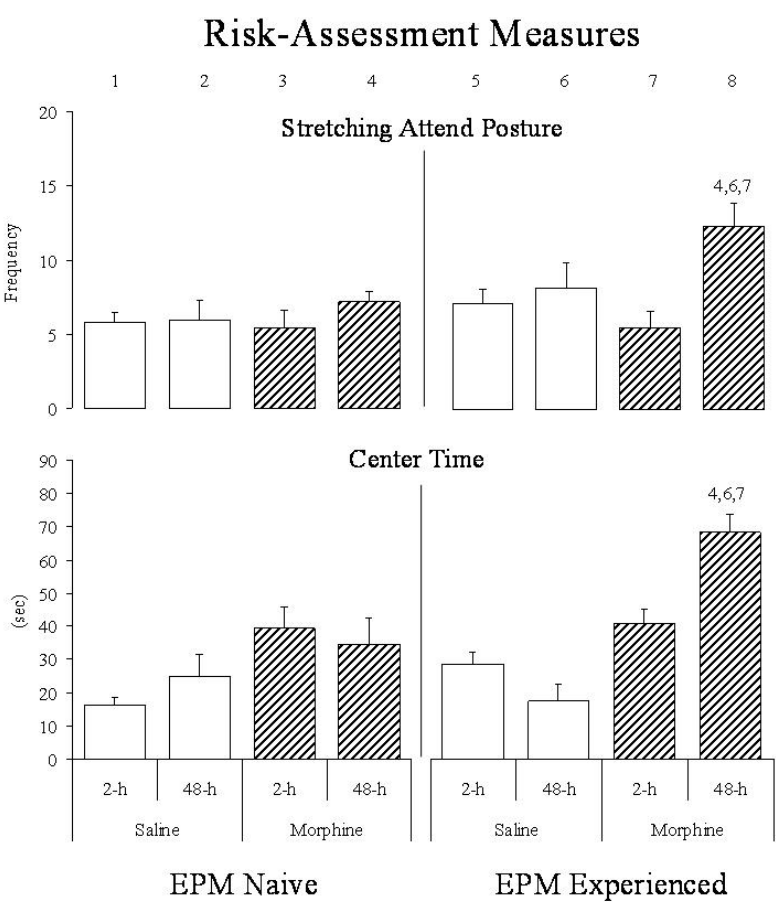

Fig. 2

Figure 2. Histograms represent the Mean \pm SEM of the frequency of stretching-attending postures (SAP) (top) and the time spent in the center of EPM (bottom), taken as measures of RiskAssessment behavior of rats not exposed to (EPM-naïve - left) or with previous experience (EPM-experienced - right) in the EPM, confined daily, during 10 days, to the distal parts of the open-arms immediately after saline or morphine s.c. injections. The animals were tested 2- (2-h) or 48-hours (48-h) after the last injection. Three-way factorial ANOVA were carried-out, followed, when appropriate, by post-hoc Tukey HSD test; $p \leq .05$. As described above, each column received a number (1 to 8 ) in order to depict the significant differences, as observed above histograms.

closed-arm entries (bottom) in the EPM by naïve and experienced saline or morphine pre-treated rats tested under the effects of morphine or during withdrawal. For the percentage of time spent in the open arms, threeway factorial ANOVA revealed significant effects of previous EPM exposure $[\mathrm{F}(1,68)=43.09, \mathrm{p}<.0001]$ and treatments $[F(1,68)=49.24, p<.0001]$. No significant effects of condition were found. However, interactive effects were obtained between treatment and plus-maze experience $[\mathrm{F}(1,68)=32.57, \mathrm{p}<.0001]$, showing that the development of place conditioning depends upon previous EPM spatial learning. Post-hoc Tukey comparisons showed that morphine increases the time spent in the open arms in rats under the effect of morphine or withdrawal. A similar result was obtained for the percentage of open-arm entries. ANOVA detected main effects of treatment $[\mathrm{F}(1,68)=30.32, \mathrm{p}<.0001]$, previous EPM experience $[\mathrm{F}(1,68)=23.68, \mathrm{p}<.0001]$ and treatment $x$ EPM skills interaction $[\mathrm{F}(1,68)=14.44$, $\mathrm{p}<.0005]$. ANOVA also showed significant effects on the number of closed-arm entries [treatment: $\mathrm{F}(1,68)=$ 10.57, $\mathrm{p}<.0005$; condition: $\mathrm{F}(1,68)=4.88, \mathrm{p}<.05]$.
Post-hoc tests revealed that this effect was a result of the increase in locomotor activity by the rats tested under the effect of morphine (2-h), which would account for the increase in the percentage of entries and time spent in the open arms observed in this group.

With regard to the risk-assessment behaviours (Figure 2), ANOVA showed significant differences among the groups in both measures, frequency of SAP [EPM previous experience: $\mathrm{F}(1,68)=4.47, \mathrm{p}$ $<.05$; condition: $\mathrm{F}(1,68)=8.46, \mathrm{p}<.005$; treatment $x$ condition interaction: $F(1,68)=4.47, p<.05]$ and time spent at the centre of the EPM [treatment: $\mathrm{F}(1,68)$ $=37.10, \mathrm{p}<.0001$; EPM previous exposure: $\mathrm{F}(1,68)$
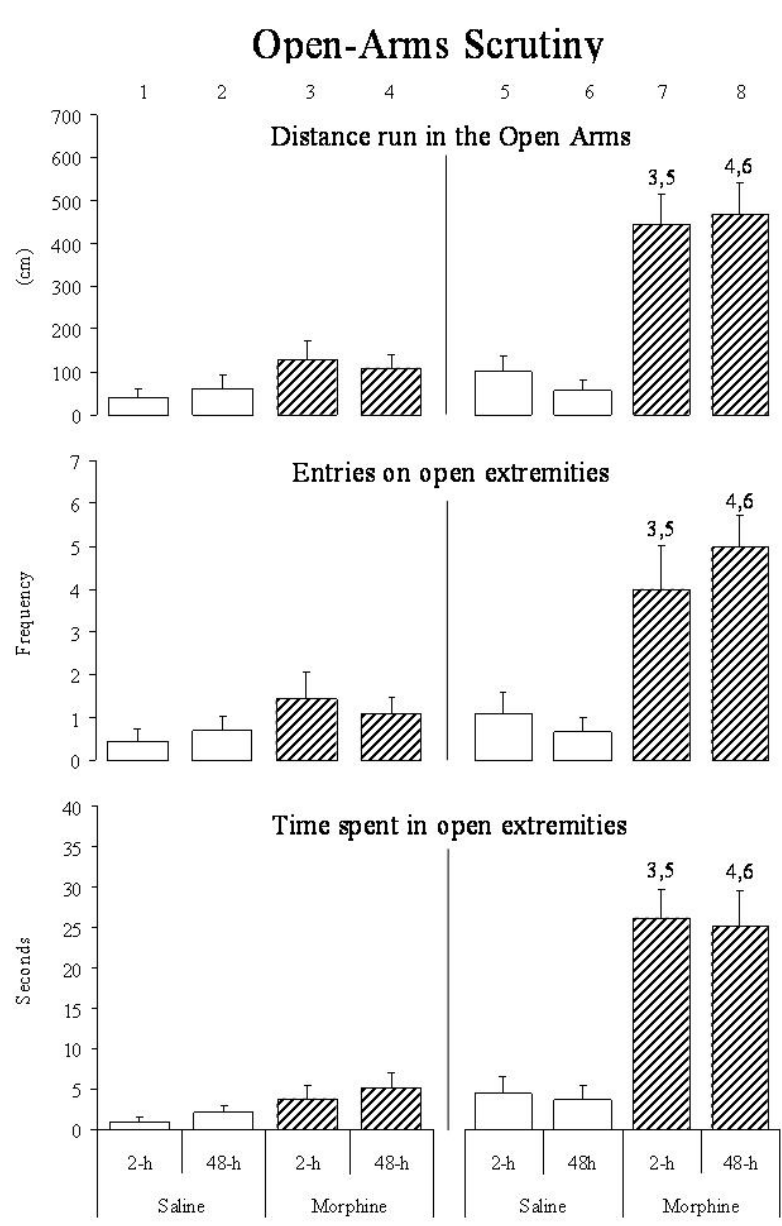

EPM Naive

EPM Experienced

Fig. 3

Figure 3. Open-arms measures, as indices of place preference, are presented as Mean $\pm \mathrm{SEM}$. Histograms represent the mean distance run in the open arms (top), and the mean frequency of entries (middle) and time spent in open extremities (bottom) of rats not exposed to (EPM Naïve - left) or with previous experience (EPM-experienced - right) in the EPM, confined daily, during 10 days, to the distal parts of the open-arms immediately after saline or morphine s.c. injections. The animals were tested 2- (2-h) or 48-hours (48-h) after the last injection. Three-way factorial ANOVA were carried-out, followed, when appropriate, by post-hoc Tukey HSD; $\mathrm{p} \leq .05$. The columns received a number ( 1 to 8 ) in order to depict the significant differences, as observed above histograms. 
$=4.26, \mathrm{p}<.05$; treatments $\mathrm{x}$ EPM skills $\mathrm{x}$ condition interaction: $F(1,68)=10.80, p<.005]$. EPM-naïve rats showed no significant differences compared to control animals in any of the measures recorded. Tukey posthoc tests showed that the differences obtained were due to significant increases in the expression of riskassessment behaviours displayed by EPM-experienced rats undergoing morphine withdrawal.

The results obtained for the open arms (Figure 3) were comparable to those previously described. Indeed, no significant results were obtained in EPM-naïve rats, regardless of the treatment given (saline or morphine) or the testing condition (2-h or 48-h). Again, plusmaze experience was necessary for the development of open-arm place preference in morphine pre-treated rats. Post-hoc tests showed that rats tested under the effect of morphine (2-h) or during drug withdrawal (48-h) increased the distance run in the open arms (top) [treatment: $\mathrm{F}(1,68)=47.41, \mathrm{p}<.0001$; EPM previous experience: $\mathrm{F}(1,68)=31.47, \mathrm{p}<.0001$; treatment $\mathrm{x}$ EPM interaction: $F(1,68)=22.68, p<.0001]$ and the number of entries (middle) [treatment: $\mathrm{F}(1,68)=28.27$, $\mathrm{p}<.0001$; EPM previous experience: $\mathrm{F}(1,68)=19.00, \mathrm{p}$ $<.0001$; treatment $x$ EPM interaction: $\mathrm{F}(1,68)=12.90, \mathrm{p}$ $<.001]$ and time spent in open-arm extremities (bottom) [treatment: $\mathrm{F}(1,68)=48.71, \mathrm{p}<.0001 ; \mathrm{EPM}$ previous experience: $\mathrm{F}(1,68)=45.90, \mathrm{p}<.0001$; treatment $\mathrm{x}$ EPM interaction: $F(1,68)=28.39, \mathrm{p}<.001]$.

\section{Discussion}

In this study we report four main findings. First, morphine pre-treated rats, naïve in respect to the overall EPM context, did not develop place preference; they behaved like control rats. Second, rats that had previous experience in the EPM showed no changes in open-arm avoidance on a second exposure when compared to naïve rats for this condition. Third, previous spatial learning of the EPM contextual cues was, in fact, a requirement for anxiety-inducing place preference for the openarms in morphine-withdrawn rats. Finally, conditioned place preference was achieved both in rats under the effect of morphine and those in withdrawal, probably through the influence of the positive (pleasurable-like $\mathrm{x}$ context) or negative (anxiety-provoking $\mathrm{x}$ context) reinforcing effects promoted by the presence or absence of the drug in the central nervous system. The former is related to the ability of the drug to function as a positive reinforcer. With regard to the later, after the interruption of chronic exposure, behaviour is guided by the negative reinforcing properties elicited by withdrawal.

Drug-induced preference for a specific place cuing for drug availability over a neutral place, i.e., the conditioned place preference, has long been used as a behavioural paradigm indicative of drug-associated memories (Sanchis-Segura \& Spanagel, 2006). The motivational changes associated with continuous drug use result, at least in part, from the formation of memories associated with drug consumption (Cannon \& Bseikri, 2004). In our study, we found that familiarity with the plus-maze cues was necessary for the development of place preference. In this case, it is likely that, during conditioning, animals not previously exposed to the EPM remain completely naïve in respect to EPM cues, except for the place in which they were confined during conditioning, i.e., the distal parts of the open-arms. In this case, the drug-associated memory formed through repeated drug exposure, which motivates the animals to approach the positive reinforcing environment, could not be evoked.

Regarding the effects obtained in rats previously exposed to the EPM, the results obtained here do not match those observed in previous studies, which claim that a second exposure to the EPM enhances open-arm avoidance, mainly through a shift from an unconditioned to a conditioned form of fear response (Carobrez \& Bertoglio, 2005; Cruz-Morales, Santos, \& Brandao, 2002; File, 1993; File \& Zangrossi, 1993; File et al., 1993; Pellow et al., 1985;). However, some particular characteristics of our study can account for these results. It was hypothesized that when the EPM is used to detect an extrinsic anxiogenic state, the anxiogenic response cannot be modified or antagonized by benzodiazepines (File, 1993). On the other hand, a simple exposure to cat odour resulted in a generalized anxiety response that was easily detected in the plus-maze (Zangrossi \& File, 1993), an anxiogenic effect counteracted by the benzodiazepine chlordiazepoxide even in plus-maze-experienced rats (File \& Zangrossi, 1993). Similarly, the study of Bertoglio and Carobrez (2002) showed that the shortacting benzodiazepine midazolam retains its efficacy in rats confined either to open or enclosed arms compared with rats naïve for the test, showing that EPM cues that induce anxiety retain their efficacy in EPM-experienced rats. In the present study, it is likely that, notwithstanding the EPM-experienced rats that had previously been submitted to the plus-maze, the intense fear generated by daily confinement in the open-arms actually prevented the anxiety generated by the EPM cues themselves. The temporal factor could also account for the results because at least 10 days elapsed between the baseline and test exposures. In this case, 10 days of exposure to the open-arms, associated with a 10-day interval between the baseline and test sessions, might mitigate the initial influence of EPM aversive cues on behaviour.

Addiction to opiates is frequently associated with the repetitive intake of large amounts concomitant with the development of physical dependence. Although the current definitions of addiction emphasize its behavioural aspects, such as compulsiveness and drug-seeking behaviour, opiate addiction is mainly defined by the presence of physical dependence (Koob, Maldonado, \& Stinus, 1992; Maldonado et al., 1992; Schulteis, Heyser, 
\& Koob, 1997; Schulteis, Markou, Gold, Stinus, \& Koob, 1994; Schulteis, Yackey, Risbrough, \& Koob, 1998; Stinus, Caille, \& Koob, 2000). Nonetheless, in a previous study we showed that low doses of morphine, which characteristically promoted slight physical symptoms in morphine-withdrawn rats, elicited intense unconditioned fear reactions (Ávila et al., 2008). In our study, morphine itself was unable to promote the locomotor activation that is commonly observed after acute injection in rats naïve for the plus-maze. Indeed, none of the measures were altered, showing that morphine did not change anxiety levels, ambulation, exploratory activity, or risk-assessment behaviour in these animals despite a marginally significant difference in the number of closed-arm entries. However, even in this case, rats under the effects of morphine presented overall fear reactions similar to those observed in control rats.

The most important results obtained in the present study concern the effects of morphine and its withdrawal in rats with prior experience in the EPM, confined to the distal parts of the open arms immediately after the s.c. drug injections. When the EPM-experienced rats were tested under the effects of morphine, an increase in the conventional and open-arm scrutiny measures was observed, similar to that noted in morphine-withdrawn rats. This probably resulted from psychomotor activation associated with place conditioning induced by morphine positive-reinforcing effects, since the observed riskassessment behaviour did not differ significantly from those obtained in withdrawn animals. In any case, highly addictive drugs, including amphetamine, cocaine, and morphine, have the ability to increase motor behaviour, an effect mainly attributed to the increase in dopamine transmission in the striatal complex (Di Chiara \& Imperato, 1988; Wise \& Bozarth, 1987).

It is well known that an increase in the frequency of SAP is a reliable index of anxiety in rodents. Fernández Espejo (1997) showed that SAP is one of the best indicators of anxiety in the EPM. In addition, Grewal, Shepherd, Bill, Fletcher, and Dourish (1997) established this response as an important component of the riskassessment repertoire of defensive behaviour in rodents. The time spent at the centre of the EPM as a reflection of anxiety-like behaviour has been discussed in less detail. However, in spite of the fact that this measure is infrequently recorded in the plus-maze, some authors have argued that the time spent at the centre of the EPM is possibly related to approach-avoidance conflict (Cruz et al., 1992; Rodgers, Lee, \& Sheperd, 1992; Trullas \& Skolnick, 1993), in a way similar to SAP. That is the reason why, in our study, both SAP and the time spent at the centre of the maze were grouped and taken as indices of risk-assessment behaviours.

The probability of facilitating drug addiction in humans has been strongly related to the existence of multiple comorbid diagnoses, such as depression and anxiety disorders (Nadal, Rotllant, Marquez, \& Armario, 2005). However, the role of the negative affective states, elicited during opiate withdrawal in rats, and negative-reinforcing properties that induce relapse, have not been widely studied. In a previous study we showed that rats withdrawn from the benzodiazepine diazepam promptly displayed drug-seeking behaviour when tested in a place-preference procedure even when, during conditioning, drug delivery was preceded by an aversive event (Souza-Pinto, Castilho, Brandao, \& Nobre, 2007). In the present study, rats experiencing the positive reinforcing effects of low doses of morphine were challenged, in a conflict paradigm, with the aversive cues of the open arms of the EPM during the place conditioning procedure. In connection with this point, opiate dependence, when produced by progressive and high doses of morphine, promotes extreme physical signs of withdrawal (Frederickson \& Pinsky, 1975; Wang, Zhao, Xiang, Wang, \& Wu, 2004) that could mask some overt behavioural elements that are commonly used as indices of fear and anxiety, such as the measures obtained with the use of the EPM. In the present study, we showed that rats withdrawn from low doses of morphine acquired strong place preference for the region of the maze where they had experienced the positive reinforcing effects of the drug, despite a continuous degree of aversion, as revealed by the increase in the frequency of SAP and in the time spent at the centre of the maze. Thus, we argue that previous spatial learning in the EPM in morphine pre-treated rats is a necessary condition for the development of relapse. In other words, overall familiarity with the context in which conditioning takes place is required for the development of place preference. This phenomenon was observed in animals tested under the effect of morphine and those withdrawn from this drug, leading us to suppose that place preference conditioning in both groups was achieved through different processes. When under the effect of morphine, rats which showed anxiety levels similar to controls probably acquired place preference due to the positive reinforcing effects of the drug. On the other hand, morphine-withdrawn rats acquired a preference for the place where they were conditioned through the influence of the negative reinforcing effects of withdrawal.

\section{Acknowledgements}

This work was supported by a grant from FAPESP (2004/02859-0).

\section{References}

Ávila, M.A.V., Ruggiero, R.N., Cabral, A., Brandão, M.L., Nobre, M.J., Castilho, V.M. (2008). Involvement of the midbrain tectum in the unconditioned fear promoted by morphine withdrawal. European Journal of Pharmacology, 590(1-3), 217-223. 
Bertoglio, L.J., \& Carobrez, A.P. (2002). Prior maze experience required to alter midazolam effects in rats submitted to the elevated plus-maze. Pharmacology Biochemistry and Behavior, 72, 449-455.

Bozarth, M.A., \& Wise, A. (1984). Anatomically distinct opioid receptor fields mediate reward and physical dependence. Science, 224, 516-517.

Cannon, C.M., \& Bseikri, M.R. (2004). Is dopamine required for natural reward? Physiology and Behavior, 81, 741-748.

Carobrez, A.P., \& Bertoglio, L. J. (2005). Ethological and temporal analyses of anxiety-like behavior: The elevated plus-maze model 20 years on. Neuroscience and Biobehavioral Reviews, 29, 1193-1205.

Castilho, V.M., Borelli, K.G., Brandao, M.L., \& Nobre, M.J. (2008). Anxiety-like symptoms induced by morphine withdrawal may be due to the sensitization of the dorsal periaqueductal grey. Physiology and Behavior, 94, 552-562.

Cruz, A.P., Frei, F., \& Graeff, F.G. (1994). Ethopharmacological analysis of rat behavior on the elevated plus-maze. Pharmacology Biochemistry and Behavior, 49, 171-176.

Cruz-Morales, S.E., Santos, N.R., \& Brandao, M.L. (2002). One-trial tolerance to midazolam is due to enhancement of fear and reduction of anxiolytic-sensitive behaviors in the elevated plus-maze retest in the rat. Pharmacology Biochemistry and Behavior, 72, 973-978.

Di Chiara, G., \& Imperato, A. (1988). Drugs abused by humans preferentially increase synaptic dopamine concentrations in the mesolimbic system of freely moving rats. Proceedings of the National Academy of Sciences U S A, 85, 5274-5278.

Fernández Espejo, E. (1997). Structure of the mouse behaviour on the elevated plus-maze test of anxiety. Behavioural Brain Research, $86,105-112$.

File, S.E. (1993). The interplay of learning and anxiety in the elevated plus-maze. Behavioural Brain Research, 58, 199-202.

File, S.E., \& Zangrossi Jr., H. (1993). One-trial tolerance to the anxiolytic actions of benzodiazepines in the elevated plus-maze, or the development of a phobic state? Psychopharmacology, 110, 240-244.

File, S.E., Zangrossi Jr., H., Viana, M., \& Graeff, F.G. (1993). Trial 2 in the elevated plus-maze: A different form of fear? Psychopharmacology, 111, 491-494.

Frederickson, R.C., \& Pinsky, C. (1975). Effects of cholinergic and anticholinergic drugs and a partial cholinergic agonist on the development and expression of physical dependence on morphine in rat. Journal of Pharmacology and Experimental Therapeutics, 193, 44-55.

Gellert, V.F., \& Holtzman, S.G. (1978). Development and maintenance of morphine tolerance and dependence in the rat by scheduled access to morphine drinking solutions. Journal of Pharmacology and Experimental Therapeutics, 205, 536-546.

Grewal, S.S., Shepherd, J.K., Bill, D.J., Fletcher, A., \& Dourish, C.T. (1997). Behavioural and pharmacological characterization of the canopy stretched attend posture test as a model of anxiety in mice and rats. Psychopharmacology, 133, 29-38.

Himmelsbach, C.K. (1943). Can the euphoric, analgesic, and physical dependence effects of drugs be separated? IV. With reference to physical dependence. Federal Proceedings, 2, 201-203.

Holmes, A., \& Rodgers, R.J. (1999). Influence of spatial and temporal manipulations and the anxiolytic efficacy of chlordiazepoxide in mice previously exposed to the elevated plus-maze. Neuroscience and Biobehavioral Reviews, 23, 971-980.

Koob, G., \& Kreek, M.J. (2007). Stress, deregulation of drug reward pathways, and the transition to drug dependence. American Journal of Psychiatry, 164, 1149-1159.

Koob, G.F., Maldonado, R., \& Stinus, L. (1992). Neural substrates of opiate withdrawal. Trends in Neuroscience, 15, 186-191.
Maldonado, R., Stinus, L., Gold, L.H., \& Koob, G.F. (1992). Role of different brain structures in the expression of the physical morphine withdrawal syndrome. Journal of Pharmacology and Experimental Therapeutics, 261, 669-677.

Nadal, R., Rotllant, D., Marquez, C., \& Armario, A. (2005). Perseverance of exploration in novel environments predicts morphine place conditioning in rats. Behavioural Brain Research, 165, 72-79.

Papaleo, F., \& Contarino, A. (2006). Gender- and morphine doselinked expression of spontaneous somatic opiate withdrawal in mice. Behavioural Brain Research, 170, 110-118.

Pellow, S., Chopin, P., File, S.E., \& Briley, M. (1985). Validation of open-closed arm entries in an elevated plus-maze as a measure of anxiety in the rat. Journal of Neuroscience Methods, 14, 149-167.

Rodgers, R.J., Lee, C., \& Sheperd, J.K. (1992). Effects of diazepam on behavioural and antinociceptive responses to the elevated plusmaze in mice depend upon treatment regimen and prior maze experience. Psychopharmacology, 106, 102-110.

Rodgers, R.J., \& Johnson, N.J.T.(1995). Factor analysis of spatiotemporal and ethological measures in the murine elevated plus-maze test of anxiety. Pharmacology Biochemistry and Behavior, 52, 297-303.

Salome, N., Salchner, P., Viltart, O., Sequeira, H., Wigger, A., Landgraf, R., \& Singewald, N. (2004). Neurobiological correlates of high (HAB) versus low anxiety-related behavior (LAB): Differential Fos expression in HAB and LAB rats. Biological Psychiatry, 55, 715-723.

Sanchis-Segura, C., \& Spanagel, R. (2006). Behavioural assessment of drug reinforcement and addictive features in rodents: An overview. Addiction Biology 11, 2-38

Schulteis, G., Heyser, C.J., \& Koob, G.F. (1997). Opiate withdrawal signs precipitated by naloxone following a single exposure to morphine: Potentiation with a second morphine exposure. Psychopharmacology, 129, 56-65.

Schulteis, G., Markou, A., Gold, L.H., Stinus, L., \& Koob, G.F. (1994). Relative sensitivity to naloxone of multiple indices of opiate withdrawal: A quantitative dose-response analysis. Journal of Pharmacology and Experimental Therapeutics, 271, 1391-1398.

Schulteis, G., Yackey, M., Risbrough, V., \& Koob, G.F. (1998). Anxiogenic-like effects of spontaneous and naloxone-precipitated opiate withdrawal in the elevated plus-maze. Pharmacology Biochemistry and Behavior, 60, 727-731.

Souza-Pinto, L.F., Castilho, V.M., Brandao, M.L., \& Nobre, M.J. (2007). The blockade of AMPA-kainate and NMDA receptors in the dorsal periaqueductal gray reduces the effects of diazepam withdrawal in rats. Pharmacology Biochemistry and Behavior, 87, 250-257.

Stinus, L., Caille, S., \& Koob, G.F. (2000). Opiate withdrawal-induced place aversion lasts for up to 16 weeks. Psychopharmacology, 149, 115-120.

Trullas, R., \& Skolnick, P. (1993). Differences in fear motivated behavior among inbred mouse strains. Psychopharmacology, 111, 323-331.

Wang, H.L., Zhao, Y., Xiang, X.H., Wang, H.S., \& Wu, W.R. (2004). Blockade of ionotropic glutamatergic transmission in the ventral tegmental area attenuates the physical signs of morphine withdrawal in rats. Progress in Neuro-Psychopharmacology and Biological Psychiatry 28, 1079-1087.

Wise, R.A. (2002). Brain reward circuitry: Insights from unsensed incentives. Neuron, 36, 229-240.

Wise, R.A., \& Bozarth, M.A. (1987). A psychomotor stimulant theory of addiction. Psychological Review, 94, 469-492.

Zangrossi Jr., H., \& File, S.E. (1993). Behavioural consequences in animal tests of anxiety and exploration of exposure to cat odor. Brain Research Bulletin, 111, 491-494. 